\title{
Grad-div stabilization for the time-dependent Boussinesq equations with inf-sup stable finite elements
}

\author{
Javier de Frutos* $\quad$ Bosco García-Archilla ${ }^{\dagger} \quad$ Julia Novo ${ }^{\ddagger}$
}

\begin{abstract}
In this paper we consider inf-sup stable finite element discretizations of the evolutionary Boussinesq equations with a grad-div type stabilization. We prove error bounds for the method with constants independent on the Rayleigh numbers
\end{abstract}

Keywords Boussinesq equations; inf-sup stable finite element methods; grad-div stabilization; High Rayleigh number flows

\section{Introduction}

Let $\tilde{\Omega} \subset \mathbb{R}^{d}, d \in\{2,3\}$, be a convex bounded domain with polyhedral and Lipschitz boundary $\partial \tilde{\Omega}$. We consider weakly non-isothemal flows following the OberbeckBoussinesq approximation for the the density $\left(\rho / \rho_{0}\right)=1-\alpha\left(\tilde{\mathcal{T}}-\tilde{\mathcal{T}}_{0}\right)$. where $\alpha$ is the thermal expansion coefficient and $\rho_{0}$ the density at reference temperature $\tilde{\mathcal{T}}_{0}$, which in this paper we take as the minimum value of the temperature $\tilde{\mathcal{T}}$ on $\partial \tilde{\Omega}$. The governing equations are

$$
\begin{aligned}
\tilde{\boldsymbol{u}}_{\tilde{t}}-\nu \Delta \tilde{\boldsymbol{u}}+(\tilde{\boldsymbol{u}} \cdot \nabla) \tilde{\boldsymbol{u}}+\nabla \tilde{p}+\alpha \boldsymbol{g}\left(\tilde{\mathcal{T}}-\tilde{\mathcal{T}}_{0}\right) & =\tilde{\boldsymbol{f}}_{\tilde{u}} \\
\nabla \cdot \tilde{\boldsymbol{u}} & =0, \\
\tilde{\mathcal{T}}_{\tilde{t}}-\kappa \Delta \tilde{\mathcal{T}}+\tilde{\boldsymbol{u}} \cdot \nabla \tilde{\mathcal{T}} & =\tilde{f}_{\tilde{\mathcal{T}}}
\end{aligned}
$$

together with initial and boundary conditions. Here $\tilde{\boldsymbol{u}}$ denotes the velocity, $\nu>0$ the kinematic viscosity, $\kappa>0$ the thermal diffusivity, $\boldsymbol{g}=-g \boldsymbol{e}_{d}$, the acceleration due to gravity, $\boldsymbol{e}_{1}, \ldots, \boldsymbol{e}_{d}$ being the coordinate vectors in $\mathbb{R}^{d} ; \tilde{\boldsymbol{f}}_{\tilde{u}}$ are other external

accelerations and $\tilde{f}_{\tilde{\mathcal{T}}}$ is the rate of production of temperature due to external sources

\footnotetext{
*Instituto de Investigación en Matemáticas (IMUVA), Universidad de Valladolid, Spain. Research supported by Spanish MINECO under grant MTM2016-78995-P (AEI) and by Junta de Castilla y León under grant VA024P17 and VA105G18 cofinanced by FEDER funds (frutos@mac.uva.es)

†Departamento de Matemática Aplicada II, Universidad de Sevilla, Sevilla, Spain. Research supported by Spanish MINECO under grant MTM2015-65608-P (bosco@esi.us.es)

${ }_{\ddagger}^{\ddagger}$ Departamento de Matemáticas, Universidad Autónoma de Madrid. Research supported by Spanish MINECO under grant MTM2016-78995-P (AEI) and by Junta de Castilla y León under grant VA024P17 cofinanced by FEDER funds (julia.novo@uam.es)
} 
of heat. The function $\tilde{p}$ is given by $\tilde{p}=\left(\tilde{\tilde{p}}+\rho_{0} g \tilde{z}\right) / \rho$, where $\tilde{\tilde{p}}$ is the pressure and $-\rho_{0} g \tilde{z}$ the hydrostatic pressure. For simplicity we only consider no slip boundary conditions for the velocity

$$
\tilde{\boldsymbol{u}}=0, \quad \text { on } \partial \Omega,
$$

and for the temperature, both prescribed values and and no flux,

$$
\tilde{\mathcal{T}}=\tilde{\mathcal{T}}_{b}, \quad \text { on } \partial \tilde{\Omega}_{D}, \quad \nabla \tilde{\mathcal{T}} \cdot \tilde{\boldsymbol{n}}=0, \quad \text { on } \partial \tilde{\Omega}_{N},
$$

where $\tilde{\boldsymbol{n}}$ denotes the exterior unit normal vector and $\partial \tilde{\Omega}=\partial \tilde{\Omega}_{D} \cup \partial \tilde{\Omega}_{N}$.

In this paper we consider inf-sup stable mixed finite element approximations to the model (2) with grad-div stabilization. Our aim is to prove error bounds with constants independent on inverse powers of the viscosity $\nu$ and the thermal diffusivity $\kappa>0$, or independent of Prandl and Rayleigh numbers (see (3) below). To this end we add to the Galerkin formulation a grad-div stabilization term. In [10] error bounds for the time-dependent Navier-Stokes equations with only grad-div stabilization and constants independent on inverse powers of the viscosity are obtained. The idea is to extend those bounds to the natural convection flow described by (2). The role of the grad-div stabilization term is explained in detail in the error analysis in the present paper (see Remark 1 below).

There are several related works that should be mentioned. In [9] the authors prove error bounds for inf-sup stable mixed finite element approximations to the model (2) with both grad-div stabilization and local projection stabilization (LPS) of streamline-upwind (SUPG)-type for both the velocity and the temperature. The authors prove bounds for the velocity and the temperature with constants independent on inverse powers of $\nu$ and $\kappa$. The main drawback of [9] is that for proving the error bounds of the method the assumption $\left\|\nabla \boldsymbol{u}_{h}\right\|_{\infty}$ bounded ( $\boldsymbol{u}_{h}$ being the approximation to the velocity) is needed and no proof for this a priori bound is included. In [10] weaker assumptions (norms $\left\|\boldsymbol{u}_{h}\right\|_{\infty}$ and $\left\|\nabla \cdot \boldsymbol{u}_{h}\right\|_{L^{2 d /(d-1)}}$ bounded) are needed to get the error bounds for the pressure once the bounds for the velocity are already obtained. However in [10] these a priori bounds are proved. Let us observe that bounds on the velocity are obtained in [10] whenever finite elements other than linears are used for the velocity in the case $d=3$ so that the assumption $\left\|\nabla \boldsymbol{u}_{h}\right\|_{\infty}$ bounded for $h \rightarrow 0$ appearing in [9] may not be valid for lower order finite elements. Error bounds for the pressure are missing in reference [9]. In this paper we get error bounds for the velocity, temperature and the pressure, without assuming a priori bounds for the velocity approximations and with only grad-div stabilization. The rate of convergence we prove is the same obtained in [9] for a scheme that apart from grad-div stabilization includes LPS-SUPG stabilization for the velocity and the temperature. In [9], also numerical experiments are presented. The authors notice that the grad-div term with an $O(1)$ parameter is essential to enforce mass conservation and to obtain good numerical results. In [15] the authors consider grad-div stabilized approximations to the model problem (2) with stabilized equal order discontinuous Galerkin methods. In this reference no error bounds are proved for the method. In agreement with the results in [9], the numerical experiments in [15] show the benefits of the increment in the mass conservation due to grad-div stabilization. Finally, in reference [5] an LPS finite element method is applied to time dependent Boussinesq equations. Several terms are added to stabilize the standard Galerkin approximation including LPS stabilization of the convective terms in 
the velocity and temperature equations and LPS grad-div stabilization. In [5] the weak convergence of the method is obtained but no error bounds for the method are proved. In particular, since no bounds are proved for the rate of convergence of the method the question of the dependence on the constants in the bounds on the Rayleigh numbers is not considered. Our aim in the present paper is, on the one hand, proving error bounds for the methods with constants that do not deteriorate for increasing values of the Rayleigh numbers and, on the other hand, doing this with the fewest possible stabilization terms. As a consequence, error bounds are obtained with constants independent on inverse powers of $\nu$ and $\kappa$ in formulation (2) with only grad-div stabilization.

\section{Preliminaries and notation}

We consider the following nondimensional form of (1) (see e. g. [17])

$$
\begin{aligned}
\boldsymbol{u}_{t}-\frac{\operatorname{Pr}}{\sqrt{\operatorname{RaPr}}} \Delta \boldsymbol{u}+(\boldsymbol{u} \cdot \nabla) \boldsymbol{u}+\nabla p & =\theta \boldsymbol{e}_{d}+\boldsymbol{f}_{\boldsymbol{u}}, \\
\nabla \cdot \boldsymbol{u} & =0, \\
\theta_{t}-\frac{1}{\sqrt{\mathrm{RaPr}}} \Delta \theta+\boldsymbol{u} \cdot \nabla \theta+\boldsymbol{c} \cdot \boldsymbol{u} & =f_{\theta},
\end{aligned}
$$

where the Prandl number Pr and Rayleigh number Ra are given by

$$
\operatorname{Pr}=\frac{\nu}{\kappa}, \quad \operatorname{Ra}=\frac{\alpha g l^{3}\left(\tilde{\mathcal{T}}_{1}-\tilde{\mathcal{T}}_{0}\right)}{\nu \kappa},
$$

$l$ being a characteristic length (for example, the diameter of $\tilde{\Omega}$ or an appropriate fraction of it) and $\tilde{\mathcal{T}}_{1}$ the maximum value of the the temperature. Equations $(2)$ are obtained from $(1)$ by taking as temperature scale $\tilde{\mathcal{T}}_{1}-\tilde{\mathcal{T}}_{0}$, and as time and velocity scales $\tilde{t}_{0}$ and $\tilde{v}_{0}$, respectively, given by

$$
\tilde{t}_{0}=\frac{l^{2}}{\kappa \sqrt{\mathrm{RaPr}}}, \quad \tilde{v}_{0}=\frac{l}{t_{0}}=\frac{\kappa}{l} \sqrt{\mathrm{RaPr}} .
$$

toghether with expressing the scaled temperature as

$$
\frac{\tilde{\mathcal{T}}-\tilde{\mathcal{T}}_{0}}{\tilde{\mathcal{T}}_{1}-\tilde{\mathcal{T}}_{0}}=\theta+\theta_{b}
$$

where $\theta_{b}$ is the conductive state in the absence of heat sources, that is, the solution of

$$
-\Delta \theta_{b}=0, \quad \text { in } \Omega, \quad \theta_{b}=\frac{\tilde{\mathcal{T}}-\tilde{\mathcal{T}}_{0}}{\tilde{\mathcal{T}}_{1}-\tilde{\mathcal{T}}_{0}}, \quad \text { on } \partial \Omega_{D}, \quad \nabla \theta_{b} \cdot \boldsymbol{n}=0, \quad \text { on } \partial \Omega_{N},
$$

so that $\theta$ satisfies homogeneous boundary conditions

$$
\theta=0, \quad \text { on } \partial \Omega_{D}, \quad \nabla \theta \cdot \boldsymbol{n}=0, \quad \text { on } \partial \Omega_{N} .
$$

For example, in rectangular cavities of size $l_{x} \times l_{y}, \theta_{b}=-\boldsymbol{e}_{1} \cdot \boldsymbol{x} / l_{x}$ for natural convection with constant temperatures on vertical walls and isolated horizontal 
walls (e.g., [14]), or $\theta_{b}=-\boldsymbol{e}_{d} \cdot \boldsymbol{x} / l_{y}$ for Rayleigh-Bénard convection with constant temperatures in horizontal walls and isolated lateral walls. With this value of $\theta$ notice that $\boldsymbol{f}_{\boldsymbol{u}}$ and $\boldsymbol{c}$ are given by

$$
\boldsymbol{f}_{\boldsymbol{u}}=\frac{\tilde{t}_{0}}{\tilde{v}_{0}} \tilde{\boldsymbol{f}}_{\tilde{\boldsymbol{u}}}+\theta_{b} \boldsymbol{e}_{d}, \quad \boldsymbol{c}=\nabla \theta_{b}
$$

In this paper we will assume that $\mathrm{Pr}$ is of moderate size but $\mathrm{Ra} \gg 1$ in (2). This corresponds for example to the fluid being a gas similar to air (at $20^{\circ} \mathrm{C}, \alpha=$ $3.43 \times 10^{-3} 1 /{ }^{\circ} \mathrm{C}, \nu=1.51 \times 10^{-5} \mathrm{~m} / \mathrm{s}^{2}, \kappa=1.01 \times 10^{-5} \mathrm{~m} / \mathrm{s}^{2}$ ) or a liquid like water (at $20^{\circ} \mathrm{C}, \alpha=2.03 \times 10^{-4} 1 /{ }^{\circ} \mathrm{C}, \nu=1.0 \times 10^{-6} \mathrm{~m} / \mathrm{s}^{2}, \kappa=7.08 \times 10^{-6} \mathrm{~m} / \mathrm{s}^{2}$ ), so that the Prandl numbers are 0.713 for air and 7.01 for water, and, consequently, in the corresponding Rayleigh numbers the quantities $l^{3}\left(\mathcal{T}_{1}-\mathcal{T}_{0}\right)$ in ${ }^{\circ} \mathrm{C} \mathrm{m}^{3}$ are multiplied by factors of $2.07 \times 10^{8}$ and $2.78 \times 10^{8}$ for air and water respectively. That is, a difference of a few degrees between the coolest and hottest parts of the fluid implies Rayleigh numbers several orders or magnitude larger. Consequently, in the present paper we assume that the diffusion coefficients in (2), which in the rest of the paper we will denote by

$$
\varepsilon=\frac{\operatorname{Pr}}{\sqrt{\mathrm{RaPr}}}, \quad \hat{\varepsilon}=\frac{1}{\sqrt{\mathrm{RaPr}}}
$$

satisfy that $\varepsilon \ll 1$ and $\hat{\varepsilon} \ll 1$.

Throughout the paper, $W^{s, p}(D)$ will denote the Sobolev space of real-valued functions defined on the domain $D \subset \mathbb{R}^{d}$ with distributional derivatives of order up to $s$ in $L^{p}(D)$. These spaces are endowed with the usual norm denoted by $\|\cdot\|_{W^{s, p}(D)}$. If $s$ is not a positive integer, $W^{s, p}(D)$ is defined by interpolation [1. In the case $s=0$, it is $W^{0, p}(D)=L^{p}(D)$. As it is standard, $W^{s, p}(D)^{d}$ will be endowed with the product norm and, since no confusion can arise, it will be denoted again by $\|\cdot\|_{W^{s, p}(D)}$. The case $p=2$ will be distinguished by using $H^{s}(D)$ to denote the space $W^{s, 2}(D)$. The space $H_{0}^{1}(D)$ is the closure in $H^{1}(D)$ of the set of infinitely differentiable functions with compact support in $D$. For simplicity, $\|\cdot\|_{s}\left(\right.$ resp. $|\cdot|_{s}$ ) is used to denote the norm (resp. seminorm) both in $H^{s}(\Omega)$ or $H^{s}(\Omega)^{d}$. The exact meaning will be clear by the context. The inner product of $L^{2}(\Omega)$ or $L^{2}(\Omega)^{d}$ will be denoted by $(\cdot, \cdot)$ and the corresponding norm by $\|\cdot\|_{0}$. The norm of the space of essentially bounded functions $L^{\infty}(\Omega)$ will be denoted by $\|\cdot\|_{\infty}$. For vector-valued functions, the same conventions will be used as before. The norm of the dual space $H^{-1}(\Omega)$ of $H_{0}^{1}(\Omega)$ is denoted by $\|\cdot\|_{-1}$. As usual, $L^{2}(\Omega)$ is always identified with its dual, so one has $H_{0}^{1}(\Omega) \subset L^{2}(\Omega) \subset H^{-1}(\Omega)$ with compact injection.

In the sequel for simplicity we will consider problem $(2)$ with homogeneous Dirichlet boundary conditions. Using the function spaces $V=H_{0}^{1}(\Omega)^{d}$,

$$
Q=L_{0}^{2}(\Omega)=\left\{q \in L^{2}(\Omega):(q, 1)=0\right\},
$$

and $H_{0}^{1}(\Omega)$ the weak formulation of problem $(2)$ is as follows: Find $(\boldsymbol{u}, p, \theta) \in$ $V \times Q \times H_{0}^{1}(\Omega)$ such that for all $(\boldsymbol{v}, q, w) \in V \times Q \times H_{0}^{1}(\Omega)$,

$$
\begin{array}{r}
\left(\partial_{t} \boldsymbol{u}, \boldsymbol{v}\right)+\varepsilon(\nabla \boldsymbol{u}, \nabla \boldsymbol{v})+((\boldsymbol{u} \cdot \nabla) \boldsymbol{u}, \boldsymbol{v})-(\nabla \cdot \boldsymbol{v}, p)+(\nabla \cdot \boldsymbol{u}, q)-\left(\theta, v^{d}\right)=\left(\boldsymbol{f}_{u}, \boldsymbol{v}\right), \\
\left(\partial_{t} \theta, w\right)+\hat{\varepsilon}(\nabla \theta, \nabla w)+((\boldsymbol{u} \cdot \nabla) \theta, w)+(\boldsymbol{c} \cdot \boldsymbol{u}, w)=\left(f_{\theta}, w\right),
\end{array}
$$


where $v^{d}$ denotes the last component of $\boldsymbol{v}$. We will denote by

$$
H^{\operatorname{div}}=\left\{\boldsymbol{u} \in L^{2}(\Omega)^{d}|\nabla \cdot \boldsymbol{u}=0, \boldsymbol{u} \cdot \mathbf{n}|_{\partial \Omega}=0\right\}
$$

and

$$
V^{\text {div }}=\{\boldsymbol{u} \in V \mid \nabla \cdot \boldsymbol{u}=0\} .
$$

From Helmholzt decomposition we can write

$$
L^{2}(\Omega)^{d}=H^{\mathrm{div}}+\left(H^{\mathrm{div}}\right)^{\perp},
$$

where

$$
\left(H^{\mathrm{div}}\right)^{\perp}=\left\{\boldsymbol{v} \in L^{2}(\Omega)^{d} \mid \boldsymbol{v}=\nabla q, q \in H^{1}(\Omega)\right\}
$$

We will denote by $\Pi: L^{2}(\Omega)^{d} \rightarrow H^{\text {div }}$ the orthogonal projector ( known as Leray projector) that maps each function in $L^{2}(\Omega)^{d}$ onto its divergence-free part, so that $\boldsymbol{u} \in L^{2}(\Omega)^{d}$ can be decomposed as $\boldsymbol{u}=\Pi \boldsymbol{u}+\nabla q, q \in H^{1}(\Omega)$. The Stokes operator in $\Omega$ is given by

$$
A: \mathcal{D}(A) \subset H^{\text {div }} \rightarrow H^{\text {div }}, \quad A=-\Pi \Delta, \quad \mathcal{D}(A)=H^{2}(\Omega)^{d} \cap V^{\text {div }} .
$$

The following Sobolev's embedding [1] will be used in the analysis: For $1 \leq p<d / s$ let $q$ be such that $\frac{1}{q}=\frac{1}{p}-\frac{s}{d}$. There exists a positive constant $C$, independent of $s$, such that

$$
\|v\|_{L^{q^{\prime}}(\Omega)} \leq C\|v\|_{W^{s, p}(\Omega)}, \quad \frac{1}{q^{\prime}} \geq \frac{1}{q}, \quad v \in W^{s, p}(\Omega) .
$$

If $p>d / s$ the above relation is valid for $q^{\prime}=\infty$. A similar embedding inequality holds for vector-valued functions.

Let $V_{h} \subset V, Q_{h} \subset Q$ and $W_{h} \subset H_{0}^{1}(\Omega)$ be families of finite element spaces composed of piecewise polynomials containing those of degrees at most $k$ for velocity and temperature and $l$ for the pressure, that correspond to a family of partitions $\mathcal{T}_{h}$ of $\Omega$ into mesh cells $K$ with diameter $h_{K}$ and maximal contained ball of radius $\rho_{K}$ and with maximal diameter $h$, satisfying the regularity assumption

$$
\max _{K \in \mathcal{T}_{h}} \frac{h_{K}}{\rho_{K}} \leq c_{0}
$$

for some $c_{0}>1$. As a consequence of $(7)$, there exist a positive constant $C_{\text {inv }}$ such that the following inverse inequality holds for each $\boldsymbol{v}_{h} \in V_{h}$, and each $K \in \mathcal{T}_{h}$, (see e.g., [4, Theorem 3.2.6])

$$
\left\|\boldsymbol{v}_{h}\right\|_{W^{m, p}(K)} \leq C_{\mathrm{inv}} h_{K}^{n-m-d\left(\frac{1}{q}-\frac{1}{p}\right)}\left\|\boldsymbol{v}_{h}\right\|_{W^{n, q}(K)},
$$

where $0 \leq n \leq m \leq 1,1 \leq q \leq p \leq \infty$. We will further assume that the meshes are quasi-uniform so that, at the price of a larger $C_{\text {inv }}$, we can replace $h_{K}$ by $h$ and $K$ by $\Omega$ in $(8)$.

In this paper, we will only consider finite element spaces satisfying the discrete inf-sup condition,

$$
\inf _{q_{h} \in Q_{h}} \sup _{\boldsymbol{v}_{h} \in V_{h}} \frac{\left(\nabla \cdot \boldsymbol{v}_{h}, q_{h}\right)}{\left\|\nabla \boldsymbol{v}_{h}\right\|_{0}\left\|q_{h}\right\|_{0}} \geq \beta_{0},
$$


with $\beta_{0}>0$, a constant independent of the mesh size $h$. For example, for the MINI element it is $k=l=1$ and for the Hood-Taylor element one has $l=k-1$. Since error bounds for the pressure depend both on $l$ and the regularity of the pressure and we will assume $p \in H^{k}(\Omega)$ and $l \geq k-1$ (in (46) below we apply (13) with $j=k-1$ ) in the sequel the error bounds will be written depending only on $k$. Apart from this continuous pressure spaces, other mixed finite elements such as the Crouzeix-Raviart element are covered by the analysis in the present paper, since we do not assume continuity of the pressure approximation.

The space of discrete divergence-free functions is denoted by

$$
V_{h}^{\operatorname{div}}=\left\{\boldsymbol{v}_{h} \in V_{h} \mid\left(\nabla \cdot \boldsymbol{v}_{h}, q_{h}\right)=0 \quad \forall q_{h} \in Q_{h}\right\}
$$

and by $A_{h}: V_{h}^{\text {div }} \rightarrow V_{h}^{\text {div }}$ is denoted the following linear operator

$$
\left(A_{h} \boldsymbol{v}_{h}, \boldsymbol{w}_{h}\right)=\left(\nabla \boldsymbol{v}_{h}, \nabla \boldsymbol{w}_{h}\right) \quad \forall \boldsymbol{v}_{h}, \boldsymbol{w}_{h} \in V_{h}^{\text {div }} .
$$

Note that from this definition, it follows for $\boldsymbol{v}_{h} \in V_{h}^{\text {div }}$ that

$$
\left\|A_{h}^{1 / 2} \boldsymbol{v}_{h}\right\|_{0}=\left\|\nabla \boldsymbol{v}_{h}\right\|_{0}, \quad\left\|\nabla A_{h}^{-1 / 2} \boldsymbol{v}_{h}\right\|_{0}=\left\|\boldsymbol{v}_{h}\right\|_{0} .
$$

In what follows, $P_{h}, P_{h}^{0}$ and $\Pi_{h}^{\text {div }}$ denote the standard $L^{2}$ projection onto $W_{h}$, $Q_{h}$ and $V_{h}$, which satisfy the following error bounds for $m=0,1$,

$$
\begin{aligned}
\left\|\theta-P_{h} \theta\right\|_{m} \leq C h^{j+1-m}\|\theta\|_{j+1}, & \forall \theta \in H^{j+1}(\Omega) \cap H_{0}^{1}(\Omega), \quad j=0, \ldots, k, \\
\left\|q-P_{h}^{0} q\right\|_{m} \leq C h^{j+1-m}\|q\|_{j+1}, & \forall q \in Q \cap H^{j+1}(\Omega), \quad j=0, \ldots, l, \\
\left\|\left(I-\Pi_{h}^{\text {div }}\right) \boldsymbol{v}\right\|_{m} \leq C h^{j+1-m}\|\boldsymbol{v}\|_{j+1}, & \forall \boldsymbol{v} \in V^{\text {div }} \cap H^{j+1}(\Omega)^{d}, \quad j=0, \ldots, k, \quad
\end{aligned}
$$

the case $m=1$ in the three estimates above being true due to the assumption that the meshes are quasi-uniform. We denote by $I_{h}$ the Clément [7] interpolant taking value 0 on $\partial \Omega$, which, for a constant $C_{I}>0$ satisfies that

$$
\left\|\theta-I_{h} \theta\right\|_{W^{m, p}} \leq C_{I} h^{j+1-m}\|\theta\|_{W^{j+1, p}}, \quad j=0, \ldots, k, \quad m=0,1,
$$

For $k=1$ and $d=3$ we will use the the elliptic projection $\pi_{h} \theta \in W_{h}$ of a function $\theta \in H^{k+1}(\Omega) \cap H_{0}^{1}(\Omega)$ satisfying

$$
\left(\nabla \pi_{h} \theta, \nabla w_{h}\right)=\left(\nabla \theta, \nabla w_{h}\right), \quad \forall w_{h} \in W_{h},
$$

one has for $0 \leq m \leq 1$

$$
\left\|\theta-\pi_{h} \theta\right\|_{m} \leq C h^{j+1-m}\|\theta\|_{j+1}, \quad j=0, \ldots, k .
$$

And, also (see [16])

$$
\log (1 / h)^{-\bar{k}}\left\|\theta-\pi_{h} \theta\right\|_{\infty}+h\left\|\nabla\left(\theta-\pi_{h} \theta\right)\right\|_{\infty} \leq C h\|\theta\|_{W^{1, \infty}(\Omega)},
$$

where $\bar{k}=1$ if $k=1$ and $\bar{k}=0$ otherwise. Finally, following [10] we consider a special Stokes projection of the velocity field that we will denote by $\boldsymbol{s}_{h}$ defined by

$$
\left(\nabla \boldsymbol{s}_{h}, \nabla \boldsymbol{v}_{h}\right)=\left(\nabla \boldsymbol{u}, \nabla \boldsymbol{v}_{h}\right), \quad \forall \boldsymbol{v}_{h} \in V_{h}^{\text {div }} .
$$


More precisely, considering the Stokes problem

$$
\begin{aligned}
-\varepsilon \Delta \boldsymbol{v}+\nabla q & =\boldsymbol{f} & & \text { in } \Omega \\
\boldsymbol{v} & =\mathbf{0} & & \text { on } \partial \Omega \\
\nabla \cdot \boldsymbol{v} & =0 & & \text { in } \Omega
\end{aligned}
$$

with right-hand side $\boldsymbol{f}=-\epsilon \Delta \boldsymbol{u}=\boldsymbol{f}_{\boldsymbol{u}}-\partial_{t} \boldsymbol{u}-(\boldsymbol{u} \cdot \nabla) \boldsymbol{u}-\nabla p+\theta \boldsymbol{e}_{d}$ and solution $(\boldsymbol{v}, q)=(\boldsymbol{u}, 0)$ the pair $\left(\boldsymbol{s}_{h}, l_{h}\right) \in V_{h} \times Q_{h}$ is the mixed finite element approximation to this Stokes problem and satisfies the bounds

$$
\begin{aligned}
\left\|\boldsymbol{u}-\boldsymbol{s}_{h}\right\|_{0}+h\left\|\boldsymbol{u}-\boldsymbol{s}_{h}\right\|_{1} & \leq C h^{j+1}\|\boldsymbol{u}\|_{j+1}, & & 0 \leq j \leq k, \\
\left\|l_{h}\right\|_{0} & \leq C \varepsilon h^{j}\|\boldsymbol{u}\|_{j+1}, & & 0 \leq j \leq k,
\end{aligned}
$$

where the constant $C$ does not depend on $\varepsilon$, see [10]. Following [6], one can also obtain the following bound for $s_{h}$

$$
\left\|\nabla\left(\boldsymbol{u}-\boldsymbol{s}_{h}\right)\right\|_{\infty} \leq C\|\nabla \boldsymbol{u}\|_{\infty}
$$

where $C$ does not depend on $\varepsilon$.

In the sequel, we will also need $L^{\infty}$ bounds of $P_{h} \theta$ that we obtain now. Let us observe that by writing $P_{h} \theta=I_{h} \theta+\left(P_{h} \theta-I_{h} \theta\right)$, where $I_{h}$ is the standard Lagrange interpolant and applying (8),

$$
\left\|P_{h} \theta\right\|_{\infty} \leq\left\|I_{h} \theta\right\|_{\infty}+C_{\mathrm{inv}} h^{-d / 2}\left\|P_{h} \theta-I_{h} \theta\right\|_{0} \leq C\|\theta\|_{\infty}+C_{\mathrm{inv}} h^{-d / 2}\left\|P_{h} \theta-I_{h} \theta\right\|_{0},
$$

where in the last inequality we have bounded $\left\|I_{h} \theta\right\|_{\infty} \leq C\|\theta\|_{\infty}$. We write $\| P_{h} \theta-$ $I_{h} \theta\left\|_{0} \leq\right\| P_{h} \theta-\theta\left\|_{0}+\right\| \theta-I_{h} \theta\left\|_{0} \leq 2\right\| \theta-I_{h} \theta \|_{0}$ and then

$$
\begin{aligned}
\left\|I_{h} \theta-\theta\right\|_{0} & \leq\left\|I_{h} \theta-\theta\right\|_{0}^{2-d / 2}\left\|I_{h} \theta-\theta\right\|_{0}^{d / 2-1} \leq C\left(\|\theta\|_{1} h\right)^{2-d / 2}\left(\|\theta\|_{2} h^{2}\right)^{d / 2-1} \\
& =C\|\theta\|_{1}^{2-d / 2}\|\theta\|_{2}^{d / 2-1} h^{d / 2}
\end{aligned}
$$

where in the last inequality we have applied (14) with $j=0$ and $j=1$. Thus, it follows that

$$
\left\|P_{h} \theta\right\|_{\infty} \leq C\|\theta\|_{\infty}+C\|\theta\|_{1}^{2-d / 2}\|\theta\|_{2}^{d / 2-1} \leq C\|\theta\|_{d-2}^{1 / 2}\|\theta\|_{2}^{1 / 2},
$$

where in the last inequality, for $d=2$, we have applied the standard interpolation inequality $\|\theta\|_{1} \leq C\left(\|\theta\|_{0}\|\theta\|_{2}\right)^{1 / 2}$, and for $d=2,3$ we have applied Agmon's inequality [2, [8, Lemma 4.10], [11]

$$
\|\theta\|_{\infty} \leq C\|\theta\|_{d-2}^{1 / 2}\|\theta\|_{2}^{1 / 2}
$$

Arguing similarly, we also have

$$
\begin{gathered}
\left\|\pi_{h} \theta\right\|_{\infty} \leq C\|\theta\|_{d-2}^{1 / 2}\|\theta\|_{2}^{1 / 2} \\
\left\|\Pi_{h}^{\text {div }} \boldsymbol{u}\right\|_{\infty} \leq C\|\boldsymbol{u}\|_{d-2}^{1 / 2}\|\boldsymbol{u}\|_{2}^{1 / 2}, \quad\left\|\boldsymbol{s}_{h}\right\|_{\infty} \leq C\|\boldsymbol{u}\|_{d-2}^{1 / 2}\|\boldsymbol{u}\|_{2}^{1 / 2}
\end{gathered}
$$

and

$$
\left\|\nabla P_{h} \theta\right\|_{\infty} \leq C\|\theta\|_{d-1}^{1 / 2}\|\theta\|_{3}^{1 / 2}, \quad\left\|\nabla \Pi_{h}^{\mathrm{div}} \boldsymbol{u}\right\|_{\infty} \leq C\|\boldsymbol{u}\|_{d-1}^{1 / 2}\|\boldsymbol{u}\|_{3}^{1 / 2}
$$


where the estimates (24) are valid for $d=2, k \geq 1$ and for $d=3$ and $k \geq 2$ only. For this reason, for $k=1$, we will use the following estimates, which are consequence of (16) and (19).

$$
\left\|\nabla \pi_{h} \theta\right\|_{\infty} \leq C\|\theta\|_{W^{1, \infty}}, \quad\left\|\nabla \boldsymbol{s}_{h}\right\|_{\infty} \leq C\|\boldsymbol{u}\|_{W^{1, \infty}}
$$

The method we consider to approximate equations (2) with homogeneous Dirichlet boundary condition is the standard Galerkin method with grad-div stabilization that reads as follows: Find $\left(\boldsymbol{u}_{h}, p_{h}, \theta_{h}\right):(0, T] \rightarrow V_{h} \times Q_{h} \times W_{h}$ such that

$$
\begin{array}{r}
\left(\partial_{t} \boldsymbol{u}_{h}, \boldsymbol{v}_{h}\right)+\varepsilon\left(\nabla \boldsymbol{u}_{h}, \nabla \boldsymbol{v}_{h}\right)+b\left(\boldsymbol{u}_{h}, \boldsymbol{u}_{h}, \boldsymbol{v}_{h}\right)-\left(p_{h}, \nabla \cdot \boldsymbol{v}_{h}\right)+\left(\nabla \cdot \boldsymbol{u}_{h}, q_{h}\right) \\
+\mu\left(\nabla \cdot \boldsymbol{u}_{h}, \nabla \cdot \boldsymbol{v}_{h}\right)-\left(\theta_{h}, v_{h}^{d}\right)=\left(\boldsymbol{f}_{\boldsymbol{u}}, \boldsymbol{v}_{h}\right), \\
\left(\partial_{t} \theta_{h}, w_{h}\right)+\hat{\varepsilon}\left(\nabla \theta_{h}, \nabla w_{h}\right)+b\left(\boldsymbol{u}_{h}, \theta_{h}, w_{h}\right)+\left(\boldsymbol{c} \cdot \boldsymbol{u}_{h}, w_{h}\right)=\left(\boldsymbol{f}_{\theta}, w_{h}\right),
\end{array}
$$

for all $\left(\boldsymbol{v}_{h}, q_{h}, w_{h}\right) \in V_{h} \times Q_{h} \times W_{h}$, with $\boldsymbol{u}_{h}(0)=I_{h} \boldsymbol{u}_{0}$. Here, and in the rest of the paper, $v_{h}^{d}$ denotes the last component of $\boldsymbol{v}_{h}$. The trilinear form $b$ is defined by

$$
b(\boldsymbol{u}, \boldsymbol{v}, \boldsymbol{w})=(B(\boldsymbol{u}, \boldsymbol{v}), \boldsymbol{w}) \quad \forall \boldsymbol{u}, \boldsymbol{v}, \boldsymbol{w} \in H_{0}^{1}(\Omega)^{d},
$$

where,

$$
B(\boldsymbol{u}, \boldsymbol{v})=(\boldsymbol{u} \cdot \nabla) \boldsymbol{v}+\frac{1}{2}(\nabla \cdot \boldsymbol{u}) \boldsymbol{v} \quad \forall \boldsymbol{u}, \boldsymbol{v} \in H_{0}^{1}(\Omega)^{d}
$$

Notice the well-known property

$$
b(\boldsymbol{u}, \boldsymbol{v}, \boldsymbol{w})=-b(\boldsymbol{u}, \boldsymbol{w}, \boldsymbol{v}) \quad \forall \boldsymbol{u}, \boldsymbol{v}, \boldsymbol{w} \in V,
$$

such that, in particular, $b(\boldsymbol{u}, \boldsymbol{w}, \boldsymbol{w})=0$ for all $\boldsymbol{u}, \boldsymbol{w} \in V$.

Before getting the error bounds of the method we will prove the existence and uniqueness of the solution of (26)-(27). We will argue as in [12, Lemma 7.12], see also [9, Theorem 1]. We observe that (26)-(27) is a system of ordinary differential equations in which the right-hand side belongs to $L^{2}(0, T)$. In case the right-hand side is continuous in $[0, T]$ we can apply Peano theorem for existence and if not one has to resort to the Carathéodory theorem. Since on the right-hand side functions appear linearly and quadratically, the Lipschitz condition is satisfied. Then, the local existence and uniqueness in some maximal interval inside $[0, T]$ can be concluded. The existence of the solution in the time interval $[0, T]$ can be achieved proving that the solution is bounded in such interval. We bound the velocity and the temperature in the following theorem. Bounds for the pressure can be easily obtained using the inf-sup condition (9)

Theorem 1 Let $\boldsymbol{u}_{h}, \theta_{h}$ be the velocity and temperature approximations in equations (26)-27). Then, for $K$ defined in (30) the following bounds hold

$$
\begin{aligned}
& \left\|\boldsymbol{u}_{h}\right\|_{L^{\infty}\left(0, T ; L^{2}\right)}^{2}+\left\|\theta_{h}\right\|_{L^{\infty}\left(0, T ; L^{2}\right)}^{2}+2 \varepsilon\left\|\nabla \boldsymbol{u}_{h}\right\|_{L^{2}\left(0, T ; L^{2}\right)}^{2} \\
& \quad+2 \mu\left\|\nabla \cdot \boldsymbol{u}_{h}\right\|_{L^{2}\left(0, T ; L^{2}\right)}^{2}+2 \hat{\varepsilon}\left\|\nabla \theta_{h}\right\|_{L^{2}\left(0, T ; L^{2}\right)}^{2} \\
& \quad \leq e^{K T}\left(\left\|\boldsymbol{u}_{h}(0)\right\|_{0}^{2}+\left\|\theta_{h}(0)\right\|_{0}^{2}\right)+\frac{1}{K}\left(e^{K T}-1\right)\left(\left\|\boldsymbol{f}_{\boldsymbol{u}}\right\|_{L^{\infty}\left(0, T ; L^{2}\right)}^{2}+\left\|\boldsymbol{f}_{\theta}\right\|_{L^{\infty}\left(0, T ; L^{2}\right)}^{2}\right) .
\end{aligned}
$$


Proof We take in (26)-(27) $\boldsymbol{v}_{h}=\boldsymbol{u}_{h}$ and $w_{h}=\theta_{h}$. Using the skew-symmetric property (28) of the bilinear term we get

$$
\begin{aligned}
& \frac{1}{2} \frac{d}{d t}\left\|\boldsymbol{u}_{h}\right\|_{0}^{2}+\varepsilon\left\|\nabla \boldsymbol{u}_{h}\right\|_{0}^{2}+\mu\left\|\nabla \cdot \boldsymbol{u}_{h}\right\|_{0}^{2} \leq \frac{1}{2}\left\|\theta_{h}\right\|_{0}^{2}+\frac{1}{2}\left\|\boldsymbol{f}_{\boldsymbol{u}}\right\|_{0}^{2}+\left\|\boldsymbol{u}_{h}\right\|_{0}^{2}, \\
& \frac{1}{2} \frac{d}{d t}\left\|\theta_{h}\right\|_{0}^{2}+\hat{\varepsilon}\left\|\nabla \theta_{h}\right\|_{0}^{2} \leq \frac{1}{2}\|\boldsymbol{c}\|_{\infty}\left\|\boldsymbol{u}_{h}\right\|_{0}^{2}+\frac{1}{2}\|\boldsymbol{c}\|_{\infty}\left\|\theta_{h}\right\|_{0}^{2} \\
&+\frac{1}{2}\left\|\boldsymbol{f}_{\theta}\right\|_{0}^{2}+\frac{1}{2}\left\|\theta_{h}\right\|_{0}^{2} .
\end{aligned}
$$

Multiplying by 2, adding the above equations and denoting by

$$
K=2+\|\boldsymbol{c}\|_{L^{\infty}\left(0, T ; L^{\infty}\right)}, \quad a(t)=\left\|\boldsymbol{u}_{h}\right\|_{0}^{2}+\left\|\theta_{h}\right\|_{0}^{2}
$$

we obtain

$$
\frac{d}{d t} a(t)+2 \varepsilon\left\|\nabla \boldsymbol{u}_{h}\right\|_{0}^{2}+2 \mu\left\|\nabla \cdot \boldsymbol{u}_{h}\right\|_{0}^{2}+2 \hat{\varepsilon}\left\|\nabla \theta_{h}\right\|_{0}^{2} \leq K a(t)+\left\|\boldsymbol{f}_{\boldsymbol{u}}\right\|_{0}^{2}+\left\|\boldsymbol{f}_{\theta}\right\|_{0}^{2} .
$$

Multiplying by $e^{-K t}$ and integrating in time we reach

$$
\begin{aligned}
& \left\|\boldsymbol{u}_{h}(t)\right\|_{0}^{2}+\left\|\theta_{h}(t)\right\|_{0}^{2}+\int_{0}^{t} 2 \varepsilon\left\|\nabla \boldsymbol{u}_{h}(s)\right\|_{0}^{2}+2 \mu\left\|\nabla \cdot \boldsymbol{u}_{h}(s)\right\|_{0}^{2}+2 \hat{\varepsilon}\left\|\nabla \theta_{h}(s)\right\|_{0}^{2} d s \\
\leq & e^{K t}\left(\left\|\boldsymbol{u}_{h}(0)\right\|_{0}^{2}+\left\|\theta_{h}(0)\right\|_{0}^{2}\right)+\frac{1}{K}\left(e^{K t}-1\right)\left(\left\|\boldsymbol{f}_{\boldsymbol{u}}\right\|_{L^{\infty}\left(0, t ; L^{2}\right)}^{2}+\left\|\boldsymbol{f}_{\theta}\right\|_{L^{\infty}\left(0, t ; L^{2}\right)}^{2}\right),
\end{aligned}
$$

\section{Error analysis of the method}

\subsection{Error bounds for the velocity and the temperature}

Because of the estimates (24) are not valid when $k=1$ and $d=3$, we will deal with the case $k=1$ differently. Let us then define

$$
\bar{k}= \begin{cases}1, & \text { if } k=1, \\ 0, & \text { otherwise }\end{cases}
$$

and let us denote by

$$
\hat{\boldsymbol{u}}_{h}=\left\{\begin{array}{ll}
\boldsymbol{s}_{h}, & \text { if } k=1, \\
\Pi_{h}^{\text {div }} \boldsymbol{u}, & \text { otherwise, }
\end{array} \quad \hat{\theta}_{h}= \begin{cases}\pi_{h} \theta, & \text { if } k=1, \\
P_{h} \theta, & \text { otherwise }\end{cases}\right.
$$

and

$$
\boldsymbol{e}_{h}=\boldsymbol{u}_{h}-\hat{\boldsymbol{u}}_{h}, \quad \eta_{h}=\theta_{h}-\hat{\theta}_{h} .
$$

We concentrate first in the case $k \geq 2$. Then, for $\boldsymbol{v}_{h} \in V_{h}^{\text {div }}$ we get

$$
\begin{aligned}
& \left(\partial_{t} \boldsymbol{e}_{h}, \boldsymbol{v}_{h}\right)+\varepsilon\left(\nabla \boldsymbol{e}_{h}, \nabla \boldsymbol{v}_{h}\right)+b\left(\boldsymbol{u}_{h}, \boldsymbol{u}_{h}, \boldsymbol{v}_{h}\right)-b\left(\hat{\boldsymbol{u}}_{h}, \hat{\boldsymbol{u}}_{h}, \boldsymbol{v}_{h}\right)+\mu\left(\nabla \cdot \boldsymbol{e}_{h}, \nabla \cdot \boldsymbol{v}_{h}\right) \\
& -\left(\eta_{h}, v_{h}^{d}\right)=\varepsilon\left(\nabla \boldsymbol{\tau}_{1}, \nabla \boldsymbol{v}_{h}\right)+\left(\boldsymbol{\tau}_{2}, \boldsymbol{v}_{h}\right)+\left(\tau_{3}+\tau_{4}, \nabla \cdot \boldsymbol{v}_{h}\right)-\left(\tau_{5}, v_{h}^{d}\right),
\end{aligned}
$$

where

$$
\begin{aligned}
\boldsymbol{\tau}_{1} & =\boldsymbol{u}-\hat{\boldsymbol{u}}_{h}, \quad \boldsymbol{\tau}_{2}=B(\boldsymbol{u}, \boldsymbol{u})-B\left(\hat{\boldsymbol{u}}_{h}, \hat{\boldsymbol{u}}_{h}\right), \\
\tau_{3} & =p-P_{h}^{0} p, \quad \tau_{4}=\mu\left(\nabla \cdot\left(\hat{\boldsymbol{u}}_{h}-\boldsymbol{u}\right)\right), \quad \tau_{5}=\theta-\hat{\theta}_{h} .
\end{aligned}
$$


And, for $w_{h} \in W_{h}$

$$
\begin{aligned}
& \left(\partial_{t} \eta_{h}, w_{h}\right)+\hat{\varepsilon}\left(\nabla \eta_{h}, \nabla w_{h}\right)+b\left(\boldsymbol{u}_{h}, \eta_{h}, w_{h}\right)+\left(\boldsymbol{c} \cdot \boldsymbol{e}_{h}, w_{h}\right)= \\
& b\left(\boldsymbol{u}, \theta, w_{h}\right)-b\left(\boldsymbol{u}_{h}, \hat{\theta}_{h}, w_{h}\right)+\left(\boldsymbol{c} \cdot\left(\boldsymbol{u}-\hat{\boldsymbol{u}}_{h}\right), w_{h}\right)+\hat{\varepsilon}\left(\nabla \tau_{5}, \nabla w_{h}\right) .
\end{aligned}
$$

Now we will take $\boldsymbol{v}_{h}=\boldsymbol{e}_{h}$ in (32) and $w_{h}=\eta_{h}$ in (34). To bound the nonlinear term we argue as in [10] and apply the skew-symmetric property (28) to get

$$
b\left(\boldsymbol{u}_{h}, \boldsymbol{u}_{h}, \boldsymbol{e}_{h}\right)-b\left(\hat{\boldsymbol{u}}_{h}, \hat{\boldsymbol{u}}_{h}, \boldsymbol{e}_{h}\right)=b\left(\boldsymbol{e}_{h}, \hat{\boldsymbol{u}}_{h}, \boldsymbol{e}_{h}\right)+b\left(\boldsymbol{u}_{h}, \boldsymbol{e}_{h}, \boldsymbol{e}_{h}\right)=b\left(\boldsymbol{e}_{h}, \hat{\boldsymbol{u}}_{h}, \boldsymbol{e}_{h}\right)
$$

And

$$
\begin{aligned}
\left|b\left(\boldsymbol{e}_{h}, \hat{\boldsymbol{u}}_{h}, \boldsymbol{e}_{h}\right)\right| & \leq\left\|\nabla \hat{\boldsymbol{u}}_{h}\right\|_{\infty}\left\|\boldsymbol{e}_{h}\right\|_{0}^{2}+\frac{1}{2}\left\|\nabla \cdot \boldsymbol{e}_{h}\right\|_{0}\left\|\hat{\boldsymbol{u}}_{h}\right\|_{\infty}\|\| \boldsymbol{e}_{h} \|_{0} \\
& \leq\left\|\nabla \hat{\boldsymbol{u}}_{h}\right\|_{\infty}\left\|\boldsymbol{e}_{h}\right\|_{0}^{2}+\frac{\mu}{4}\left\|\nabla \cdot \boldsymbol{e}_{h}\right\|_{0}^{2}+\frac{\left\|\hat{\boldsymbol{u}}_{h}\right\|_{\infty}^{2}}{4 \mu}\left\|\boldsymbol{e}_{h}\right\|_{0}^{2} .
\end{aligned}
$$

Remark 1 Notice that the grad-div stabilization term is essential in the above error bound since the second term on the right-hand side of (35) will be absorbed into the left-hand side of the error equation thanks to the grad-div term. Also, the chosen skew-symmetric form of the nonlinear term is essential since in case one considers the standard convective form of the nonlinear term the contribution $b\left(\boldsymbol{u}_{h}, \boldsymbol{e}_{h}, \boldsymbol{e}_{h}\right)$ would be equal to $\left(\boldsymbol{u}_{h} \cdot \nabla \boldsymbol{e}_{h}, \boldsymbol{e}_{h}\right)$ instead of zero. Then, one needs to handle this term which seems not to be easy with the error analysis we apply.

Then, we get for the first error equation

$$
\begin{array}{r}
\frac{1}{2} \frac{d}{d t}\left\|\boldsymbol{e}_{h}\right\|_{0}^{2}+\frac{\varepsilon}{2}\left\|\nabla \boldsymbol{e}_{h}\right\|_{0}^{2}+\frac{\mu}{2}\left\|\nabla \cdot \boldsymbol{e}_{h}\right\|_{0}^{2} \leq\left(1+\left\|\nabla \hat{\boldsymbol{u}}_{h}\right\|_{\infty}+\frac{\left\|\hat{\boldsymbol{u}}_{h}\right\|_{\infty}^{2}}{4 \mu}\right)\left\|\boldsymbol{e}_{h}\right\|_{0}^{2} \\
+\frac{1}{2}\left\|\eta_{h}\right\|_{0}^{2}+\frac{\varepsilon}{2}\left\|\nabla \boldsymbol{\tau}_{1}\right\|_{0}^{2}+\left\|\boldsymbol{\tau}_{2}\right\|_{0}^{2}+\frac{1}{\mu}\left\|\tau_{3}+\tau_{4}\right\|_{0}^{2}+\left\|\tau_{5}\right\|_{0}^{2}
\end{array}
$$

For the second error equation, since applying (28) we get $b\left(\boldsymbol{u}_{h}, \eta_{h}, \eta_{h}\right)=0$ and then

$$
\begin{aligned}
\frac{1}{2} \frac{d}{d t}\left\|\eta_{h}\right\|_{0}^{2}+\frac{\hat{\varepsilon}}{2}\left\|\nabla \eta_{h}\right\|_{0}^{2} \leq & \left|b\left(\boldsymbol{u}, \theta, \eta_{h}\right)-b\left(\boldsymbol{u}_{h}, \hat{\theta}_{h}, \eta_{h}\right)\right|+\frac{\hat{\varepsilon}}{2}\left\|\nabla \tau_{5}\right\|_{0}^{2}+\frac{\|\boldsymbol{c}\|_{\infty}}{2}\left\|\boldsymbol{e}_{h}\right\|_{0}^{2} \\
& +\frac{\|\boldsymbol{c}\|_{\infty}}{2}\left\|\boldsymbol{u}-\hat{\boldsymbol{u}}_{h}\right\|_{0}^{2}+\|\boldsymbol{c}\|_{\infty}\left\|\eta_{h}\right\|_{0}^{2}
\end{aligned}
$$

We only need to bound the first term on the right hand side of (37). Then, we observe that

$$
b\left(\boldsymbol{u}, \theta, \eta_{h}\right)-b\left(\boldsymbol{u}_{h}, \hat{\theta}_{h}, \eta_{h}\right)=\left(\boldsymbol{u} \cdot \nabla \theta, \eta_{h}\right)-\left(\boldsymbol{u}_{h} \cdot \nabla \hat{\theta}_{h}, \eta_{h}\right)-\frac{1}{2}\left(\left(\nabla \cdot \boldsymbol{u}_{h}\right) \hat{\theta}_{h}, \eta_{h}\right) .
$$

For the first two terms on the right-hand side of (38) adding and subtracting $\hat{\boldsymbol{u}}_{h}$ and applying Sobolev inequality (6) we get

$$
\begin{aligned}
& \left(\boldsymbol{u} \cdot \nabla \theta, \eta_{h}\right)-\left(\boldsymbol{u}_{h} \cdot \nabla \hat{\theta}_{h}, \eta_{h}\right)=\left(\left(\boldsymbol{u}-\hat{\boldsymbol{u}}_{h}\right) \cdot \nabla \theta, \eta_{h}\right)+\left(\hat{\boldsymbol{u}}_{h} \cdot \nabla \tau_{5}, \eta_{h}\right)-\left(\boldsymbol{e}_{h} \cdot \nabla \hat{\theta}_{h}, \eta_{h}\right) \\
& \quad \leq\left\|\boldsymbol{u}-\hat{\boldsymbol{u}}_{h}\right\|_{L^{2 d}}\|\nabla \theta\|_{L^{2 d /(d-1)}}\left\|\eta_{h}\right\|_{0}+\left\|\hat{\boldsymbol{u}}_{h}\right\|_{\infty}\left\|\tau_{5}\right\|_{1}\left\|\eta_{h}\right\|_{0}+\left\|\boldsymbol{e}_{h}\right\|_{0}\left\|\nabla \hat{\theta}_{h}\right\|_{\infty}\left\|\eta_{h}\right\|_{0} \quad(39) \\
& \quad \leq C\left\|\boldsymbol{u}-\hat{\boldsymbol{u}}_{h}\right\|_{1}^{2}\|\nabla \theta\|_{L^{2 d /(d-1)}}^{2}+\frac{1}{2}\left\|\hat{\boldsymbol{u}}_{h}\right\|_{\infty}^{2}\left\|\tau_{5}\right\|_{1}^{2}+\left\|\eta_{h}\right\|_{0}^{2}+\frac{1}{2}\left\|\nabla \hat{\theta}_{h}\right\|_{\infty}\left(\left\|\boldsymbol{e}_{h}\right\|_{0}^{2}+\left\|\eta_{h}\right\|_{0}^{2}\right)
\end{aligned}
$$


For the third term on the right-hand side of (38) arguing similarly we reach

$$
\begin{aligned}
\left(\left(\nabla \cdot \boldsymbol{u}_{h}\right) \hat{\theta}_{h}, \eta_{h}\right) & =\left(\left(\nabla \cdot \boldsymbol{e}_{h}\right) \hat{\theta}_{h}, \eta_{h}\right)+\left(\left(\nabla \cdot\left(\hat{\boldsymbol{u}}_{h}-\boldsymbol{u}\right)\right) \hat{\theta}_{h}, \eta_{h}\right) \\
& \leq\left\|\nabla \cdot \boldsymbol{e}_{h}\right\|_{0}\left\|\hat{\theta}_{h}\right\|_{\infty}\left\|\eta_{h}\right\|_{0}+\left\|\hat{\boldsymbol{u}}_{h}-\boldsymbol{u}\right\|_{1}\left\|\hat{\theta}_{h}\right\|_{\infty}\left\|\eta_{h}\right\|_{0} \\
& \leq \frac{\mu}{2}\left\|\nabla \cdot \boldsymbol{e}_{h}\right\|_{0}^{2}+\frac{1}{2} \frac{\left\|\hat{\theta}_{h}\right\|_{\infty}^{2}}{\mu}\left\|\eta_{h}\right\|_{0}^{2}+\frac{1}{2}\left\|\hat{\boldsymbol{u}}_{h}-\boldsymbol{u}\right\|_{1}^{2}\left\|\hat{\theta}_{h}\right\|_{\infty}^{2}+\frac{\left\|\eta_{h}\right\|_{0}^{2}}{2}
\end{aligned}
$$

Inserting (39) and (40) in (38) we reach

$$
\begin{aligned}
\left|b\left(\boldsymbol{u}, \theta, \eta_{h}\right)-b\left(\boldsymbol{u}_{h}, \hat{\theta}_{h}, \eta_{h}\right)\right| \leq( & \left.\frac{5}{4}+\frac{1}{2}\left\|\nabla \hat{\theta}_{h}\right\|_{\infty}+\frac{\left\|\hat{\theta}_{h}\right\|_{\infty}^{2}}{4 \mu}\right)\left\|\eta_{h}\right\|_{0}^{2}+\frac{1}{2}\left\|\nabla \hat{\theta}_{h}\right\|_{\infty}\left\|\boldsymbol{e}_{h}\right\|_{0}^{2} \\
& +\frac{\mu}{4}\left\|\nabla \cdot \boldsymbol{e}_{h}\right\|_{0}^{2}+\left(C\|\nabla \theta\|_{L^{2 d /(d-1)}}^{2}+\frac{1}{4}\left\|\hat{\theta}_{h}\right\|_{\infty}^{2}\right)\left\|\boldsymbol{u}-\hat{\boldsymbol{u}}_{h}\right\|_{1}^{2} \\
& +\frac{1}{2}\left\|\hat{\boldsymbol{u}}_{h}\right\|_{\infty}^{2}\left\|\tau_{5}\right\|_{1}^{2} .
\end{aligned}
$$

Going back to (37) we get

$$
\begin{aligned}
\frac{1}{2} \frac{d}{d t}\left\|\eta_{h}\right\|_{0}^{2}+\frac{\hat{\varepsilon}}{2}\left\|\nabla \eta_{h}\right\|_{0}^{2} \leq( & \left.\frac{5}{4}+\|\boldsymbol{c}\|_{\infty}+\frac{1}{2}\left\|\nabla \hat{\theta}_{h}\right\|_{\infty}+\frac{\left\|\hat{\theta}_{h}\right\|_{\infty}^{2}}{4 \mu}\right)\left\|\eta_{h}\right\|_{0}^{2} \\
& +\frac{1}{2}\left(\left\|\nabla \hat{\theta}_{h}\right\|_{\infty}+\|\boldsymbol{c}\|_{\infty}\right)\left\|\boldsymbol{e}_{h}\right\|_{0}^{2} \\
& +\frac{\mu}{4}\left\|\nabla \cdot \boldsymbol{e}_{h}\right\|_{0}^{2}+\left(C\|\nabla \theta\|_{L^{2 d /(d-1)}}^{2}+\frac{1}{4}\left\|\hat{\theta}_{h}\right\|_{\infty}^{2}\right)\left\|\boldsymbol{u}-\hat{\boldsymbol{u}}_{h}\right\|_{1}^{2} \\
& +\frac{1}{2}\left\|\hat{\boldsymbol{u}}_{h}\right\|_{\infty}^{2}\left\|\tau_{5}\right\|_{1}^{2}+\frac{\hat{\varepsilon}}{2}\left\|\nabla \tau_{5}\right\|_{0}^{2}+\frac{\|\boldsymbol{c}\|_{\infty}}{2}\left\|\boldsymbol{u}-\hat{\boldsymbol{u}}_{h}\right\|_{0}^{2} .
\end{aligned}
$$

Adding equations (36) and (41) and multiplying by 2 we get

$$
\begin{aligned}
& \frac{d}{d t}\left(\left\|\boldsymbol{e}_{h}\right\|_{0}^{2}+\left\|\eta_{h}\right\|_{0}^{2}\right)+\varepsilon\left\|\nabla \boldsymbol{e}_{h}\right\|_{0}^{2}+\hat{\varepsilon}\left\|\nabla \eta_{h}\right\|_{0}^{2}+\frac{\mu}{2}\left\|\nabla \cdot \boldsymbol{e}_{h}\right\|_{0}^{2} \leq h(t)\left(\left\|\boldsymbol{e}_{h}\right\|_{0}^{2}+\left\|\eta_{h}\right\|_{0}^{2}\right) \\
& \quad+\varepsilon\left\|\nabla \boldsymbol{\tau}_{1}\right\|_{0}^{2}+2\left\|\boldsymbol{\tau}_{2}\right\|_{0}^{2}+\frac{2}{\mu}\left\|\tau_{3}+\tau_{4}\right\|_{0}^{2}+2\left\|\tau_{5}\right\|_{0}^{2}+\|\boldsymbol{c}\|_{\infty}\left\|\boldsymbol{u}-\hat{\boldsymbol{u}}_{h}\right\|_{0}^{2} \\
& \quad+\left(C\|\nabla \theta\|_{L^{2 d /(d-1)}}^{2}+\frac{1}{2}\left\|\hat{\theta}_{h}\right\|_{\infty}^{2}\right)\left\|\boldsymbol{u}-\hat{\boldsymbol{u}}_{h}\right\|_{1}^{2}+\left\|\hat{\boldsymbol{u}}_{h}\right\|_{\infty}^{2}\left\|\tau_{5}\right\|_{1}^{2}+\hat{\varepsilon}\left\|\nabla \tau_{5}\right\|_{0}^{2}
\end{aligned}
$$

where

$$
\begin{gathered}
h(t)=\max \left(2+2\left\|\nabla \hat{\boldsymbol{u}}_{h}\right\|_{\infty}+\frac{1}{2 \mu}\left\|\hat{\boldsymbol{u}}_{h}\right\|_{\infty}^{2}+\left\|\nabla \hat{\theta}_{h}\right\|_{\infty}+\|\boldsymbol{c}\|_{\infty},\right. \\
\left.\frac{7}{2}+2\|\boldsymbol{c}\|_{\infty}+\left\|\nabla \hat{\theta}_{h}\right\|_{\infty}+\frac{1}{2 \mu}\left\|\hat{\theta}_{h}\right\|_{\infty}^{2}\right) .
\end{gathered}
$$

Observe that in view of $L^{\infty}$ bounds 20 24), except when $d=3$ and $k=1$, we have that $h(t)$ is in $L^{1}(0, T)$ if $\|\theta\|_{2}$ and $\|\boldsymbol{u}\|_{2}$ are bounded and $\|\theta\|_{W^{1, \infty}}$ and $\|\boldsymbol{u}\|_{W^{1, \infty}}$ (or $\|\theta\|_{3}^{1 / 2}$ and $\left.\|\boldsymbol{u}\|_{3}^{1 / 2}\right)$ are in $L^{1}(0, T)$, which is the case if both $\theta$ and $\boldsymbol{u}$ are sufficiently regular. Let us also observe that from (20) and Sobolev inequality (6) we get for the first term on the last line of 42

$$
C\|\nabla \theta\|_{L^{2 d /(d-1)}}^{2}+\frac{1}{2}\left\|\hat{\theta}_{h}\right\|_{\infty}^{2} \leq C\left(\|\theta\|_{2}+\|\theta\|_{d-2}^{1 / 2}\|\theta\|_{2}^{1 / 2}\right) \leq C\|\theta\|_{2} .
$$


Let us denote by

$$
\begin{aligned}
a(t)= & \left\|\boldsymbol{e}_{h}\right\|_{0}^{2}+\left\|\eta_{h}\right\|_{0}^{2}, \\
b(t)= & \varepsilon\left\|\nabla \boldsymbol{e}_{h}\right\|_{0}^{2}+\hat{\varepsilon}\left\|\nabla \eta_{h}\right\|_{0}^{2}+\frac{\mu}{2}\left\|\nabla \cdot \boldsymbol{e}_{h}\right\|_{0}^{2}, \\
c(t)= & \varepsilon\left\|\nabla \boldsymbol{\tau}_{1}\right\|_{0}^{2}+2\left\|\boldsymbol{\tau}_{2}\right\|_{0}^{2}+\frac{2}{\mu}\left\|\tau_{3}+\tau_{4}\right\|_{0}^{2}+2\left\|\tau_{5}\right\|_{0}^{2}+\|\boldsymbol{c}\|_{\infty}\left\|\boldsymbol{u}-\hat{\boldsymbol{u}}_{h}\right\|_{0}^{2} \\
& \quad+\left(C\|\nabla \theta\|_{L^{2 d /(d-1)}}^{2}+\frac{1}{2}\left\|\hat{\theta}_{h}\right\|_{\infty}^{2}\right)\left\|\boldsymbol{u}-\hat{\boldsymbol{u}}_{h}\right\|_{1}^{2}+\left\|\hat{\boldsymbol{u}}_{h}\right\|_{\infty}^{2}\left\|\tau_{5}\right\|_{1}^{2}+\hat{\varepsilon}\left\|\nabla \tau_{5}\right\|_{0}^{2} .
\end{aligned}
$$

With the above notation we write $(42)$ as

$$
\frac{d}{d t} a(t)+b(t) \leq h(t) a(t)+c(t)
$$

Then denoting by

$$
K(t, s)=\int_{s}^{t} h(r) d r
$$

multiplying by the integrating factor $\exp (-K(t, 0))$ the error equation 42 we get

$$
\frac{d}{d t}\left(e^{-K(t, 0)} a(t)\right)+e^{-K(t, 0)} b(t) \leq e^{-K(t, 0)} c(t) .
$$

Integrating in time and multiplying by $\exp (K(t, 0))$ we reach

$$
\begin{aligned}
& \left\|\boldsymbol{e}_{h}\right\|_{0}^{2}+\left\|\eta_{h}\right\|_{0}^{2}+\int_{0}^{t} e^{K(t, s)}\left(\varepsilon\left\|\nabla \boldsymbol{e}_{h}\right\|_{0}^{2}+\hat{\varepsilon}\left\|\nabla \eta_{h}\right\|_{0}^{2}+\frac{\mu}{2}\left\|\nabla \cdot \boldsymbol{e}_{h}\right\|_{0}^{2}\right) d s \\
& \quad \leq e^{K(t, 0)}\left(\left\|\boldsymbol{e}_{h}(0)\right\|_{0}^{2}+\left\|\eta_{h}(0)\right\|_{0}^{2}\right) \\
& \quad+\int_{0}^{t} e^{K(t, s)}\left(\varepsilon\left\|\nabla \boldsymbol{\tau}_{1}\right\|_{0}^{2}+2\left\|\boldsymbol{\tau}_{2}\right\|_{0}^{2}+\frac{2}{\mu}\left\|\tau_{3}+\tau_{4}\right\|_{0}^{2}+2\left\|\tau_{5}\right\|_{0}^{2}+\|\boldsymbol{c}\|_{\infty}\left\|\boldsymbol{u}-\hat{\boldsymbol{u}}_{h}\right\|_{0}^{2}\right) d s \\
& \quad+\int_{0}^{t} e^{K(t, s)}\left(C\|\theta\|_{2}\left\|\boldsymbol{u}-\hat{\boldsymbol{u}}_{h}\right\|_{1}^{2}+\left\|\hat{\boldsymbol{u}}_{h}\right\|_{\infty}^{2}\left\|\tau_{5}\right\|_{1}^{2}+\hat{\varepsilon}\left\|\nabla \tau_{5}\right\|_{0}^{2}\right) d s .
\end{aligned}
$$

To conclude we only need to bound the truncation errors on the right-hand side of (43). To bound $\left\|\nabla \boldsymbol{\tau}_{1}\right\|_{0}$ we apply (13) to get

$$
\varepsilon\left\|\nabla \boldsymbol{\tau}_{1}\right\|_{0}^{2} \leq C \varepsilon h^{2 k}\|\boldsymbol{u}\|_{k+1}^{2} .
$$

For the next truncation error we argue as in [10, (38)] applying (13) to get

$$
\left\|\boldsymbol{\tau}_{2}\right\|_{0}^{2} \leq C h^{2 k}\|\boldsymbol{u}\|_{2}^{2}\|\boldsymbol{u}\|_{k+1}^{2}
$$

And for the next term applying (12) with $m=0$ and $j=k-1$ and (13) we obtain

$$
\left\|\tau_{3}+\tau_{4}\right\|_{0}^{2} \leq C h^{2 k}\left(\|p\|_{k}^{2}+\mu^{2}\|\boldsymbol{u}\|_{k+1}^{2}\right) .
$$

Finally $\left\|\boldsymbol{u}-\hat{\boldsymbol{u}}_{h}\right\|_{1} \leq C h^{k}\|\boldsymbol{u}\|_{k+1}$ and $\left\|\boldsymbol{u}-\hat{\boldsymbol{u}}_{h}\right\|_{0} \leq C h^{k}\|\boldsymbol{u}\|_{k}$ from (13) and $\left\|\tau_{5}\right\|_{j} \leq$ $C h^{k+1-j}\|\theta\|_{k+1}$ for $j=0,1$ and $\hat{\varepsilon}\left\|\nabla \tau_{5}\right\|_{0} \leq C \hat{\varepsilon} h^{k}\|\theta\|_{k+1}$ from (11).

We deal now with the case $k=1$. Here we must take $\hat{\boldsymbol{u}}_{h}=\boldsymbol{s}_{h}$ and $\hat{\theta}_{h}=\pi_{h} \theta$. Then, in the error equations (32) and (34) the terms $\varepsilon\left(\nabla \tau_{1}, \nabla \boldsymbol{v}_{h}\right)$ and $\hat{\varepsilon}\left(\nabla \tau_{5}, \nabla w_{h}\right)$ 
must be replaced by $\left(\partial_{t} \boldsymbol{\tau}_{1}, \boldsymbol{v}_{h}\right)$ and $\left(\partial_{t} \tau_{5}, w_{h}\right)$, respectively. Repeating the arguments from (34) onwards (with some obvious changes) we arrive to 43 with $h(t)$ replaced by $1+h(t)$, and $\varepsilon\left\|\nabla \boldsymbol{\tau}_{1}\right\|_{0}^{2}$ and $\hat{\varepsilon}\left\|\nabla \tau_{5}\right\|_{0}^{2}$ replaced by $\left\|\partial_{t} \boldsymbol{\tau}_{1}\right\|_{0}^{2}$ and $\left\|\partial_{t} \tau_{5}\right\|_{0}^{2}$, respectively. Notice also that

$$
\left\|\partial_{t} \boldsymbol{\tau}_{1}\right\|_{0}^{2} \leq C h^{2}\left\|\partial_{t} \boldsymbol{u}\right\|_{1}^{2}, \quad\left\|\partial_{t} \tau_{5}\right\|_{0}^{2} \leq C h^{2}\left\|\partial_{t} \theta\right\|_{1}^{2} .
$$

Notice also that, applying $(25)$ to bound $\left\|\nabla \hat{\theta}_{h}\right\|_{\infty}$ and $\left\|\nabla \hat{\boldsymbol{u}}_{h}\right\|_{\infty}$, we have that when $k=1 h(t)$ is in $L^{1}(0, T)$ under the same conditions as in the rest of the cases.

Let us also observe that for $d=2,3$ and $k \geq 1$ one has $1 \leq \exp (K(t, s)) \leq$ $\exp (L(T))$ with

$$
\begin{aligned}
L(T)=\bar{k} T+\max & \left(\int _ { 0 } ^ { T } \left(2+C\|\boldsymbol{u}\|_{W^{1, \infty}}+\frac{C}{2 \mu}\left(\|\boldsymbol{u}\|_{d-2}\|\boldsymbol{u}\|_{2}\right.\right.\right. \\
+ & C\left(\|\theta\|_{W^{1, \infty}}+\|\boldsymbol{c}\|_{L^{\infty}(\Omega)}\right) d s, \\
& \int_{0}^{T}\left(\frac{7}{2}+2\|\boldsymbol{c}\|_{L^{\infty}(\Omega)}+C\left(\|\theta\|_{W^{1, \infty}}+\frac{C}{2 \mu}\|\theta\|_{d-2}\|\theta\|_{2}\right) d s\right),
\end{aligned}
$$

where $C$ is independent of $\varepsilon$. Then, by expressing $\boldsymbol{u}_{h}-\boldsymbol{u}=\boldsymbol{e}_{h}+\hat{\boldsymbol{u}}_{h}-\boldsymbol{u}, \theta_{h}-\theta=$ $\eta_{h}-\hat{\theta}_{h}-\theta$ and recalling bounds 11 18) we arrive to the following result.

Theorem 2 For $T>0$ let us assume for the solution $(\boldsymbol{u}, p, \theta)$ of $(2)$ that

$$
\begin{gathered}
\boldsymbol{u} \in L^{2}\left(0, T, H^{k+1}(\Omega)^{d}\right) \cap L^{2}\left(0, T, W^{1, \infty}\left(\Omega^{d}\right)\right) \cap L^{\infty}\left(0, T, H^{2}(\Omega)^{d}\right), \\
\theta \in L^{2}\left(0, T, H^{k+1}(\Omega)\right) \cap L^{2}\left(0, T, W^{1, \infty}(\Omega)\right),
\end{gathered}
$$

$\boldsymbol{u}(0) \in H^{k}(\Omega), \theta(0) \in H^{k}(\Omega), \partial_{t} \boldsymbol{u} \in L^{2}\left(0, T, H^{1}(\Omega)^{d}\right), \partial_{t} \theta \in L^{2}\left(0, T, H^{1}(\Omega)\right)$ and $p \in L^{2}\left(0, T, H^{k}(\Omega)\right)$ with $k \geq 1$. Then there exists a positive constant $C$ depending on

$$
\begin{aligned}
& \max _{t \in[0, T]}\left(\|\boldsymbol{u}(t)\|_{k}^{2}+\|\theta(t)\|_{k}^{2}\right) \\
& +\int_{0}^{T}\left(\frac{\|p(t)\|_{H^{k}}^{2}}{\mu}+\bar{k}\left\|\partial_{t} \boldsymbol{u}(t)\right\|_{1}^{2}+\left((1-\bar{k}) \varepsilon+\mu+\|\boldsymbol{u}(t)\|_{2}^{2}\right)\|\boldsymbol{u}(t)\|_{k+1}^{2}\right) d t \\
& +\int_{0}^{T}\left(\left(\|\theta(t)\|_{k}^{2}+\left(\|\theta(t)\|_{2}+\|\theta(t)\|_{W^{1, \infty}(\Omega)}\right)\|\boldsymbol{u}(t)\|_{k+1}^{2}\right)+\|\boldsymbol{c}\|_{\infty}\|\boldsymbol{u}\|_{k}^{2}\right) d t \\
& +\int_{0}^{T}\left(\left(\|\boldsymbol{u}(t)\|_{2}^{2}+\|\boldsymbol{u}(t)\|_{W^{1, \infty}(\Omega)^{2}}^{2}+(1-\bar{k}) \hat{\varepsilon}\right)\|\theta(t)\|_{k+1}^{2}+\bar{k}\left\|\partial_{t} \theta(t)\right\|_{1}^{2}\right) d t
\end{aligned}
$$

but not directly on inverse powers of $\varepsilon$ and $\hat{\varepsilon}$ such that the following bound holds for $\boldsymbol{e}_{h}=\boldsymbol{u}_{h}-\hat{\boldsymbol{u}}_{h}, \eta_{h}=\theta_{h}-\hat{\theta}_{h}$ and $t \in[0, T]$

$$
\begin{aligned}
& \left\|\boldsymbol{u}_{h}(t)-\boldsymbol{u}(t)\right\|_{0}^{2}+\left\|\theta_{h}(t)-\theta(t)\right\|_{0}^{2} \\
& \quad+\int_{0}^{t}\left(\varepsilon\left\|\nabla\left(\boldsymbol{u}_{h}(s)-\boldsymbol{u}(s)\right)\right\|_{0}^{2}+\hat{\varepsilon}\left\|\nabla\left(\theta_{h}(s)-\theta(s)\right)\right\|_{0}^{2}+\mu\left\|\nabla \cdot\left(\boldsymbol{u}_{h}(s)-\boldsymbol{u}(s)\right)\right\|_{0}^{2}\right) d s \\
& \quad \leq C \exp (L(T)) h^{2 k},
\end{aligned}
$$

where $L(T)$ is defined in (48) and $\bar{k}$ in (31). 
Remark 2 Observe that due to Agmon's inequality, when $k \geq 2$ the hypotheses on $\boldsymbol{u}$ and $\theta$ in Theorem (2) can be simplified to

$$
\begin{aligned}
& \boldsymbol{u} \in L^{2}\left(0, T, H^{k+1}(\Omega)^{d}\right) \cap L^{\infty}\left(0, T, H^{2}(\Omega)^{d}\right), \\
& \theta \in L^{2}\left(0, T, H^{k+1}(\Omega)\right), p \in L^{2}\left(0, T, H^{k}(\Omega)\right) .
\end{aligned}
$$

$\boldsymbol{u}(0) \in H^{k}(\Omega), \theta(0) \in H^{k}(\Omega)$.

Remark 3 It is well known that unless the forcing term $\boldsymbol{f}_{\boldsymbol{u}}$ in $(2)$ and $\boldsymbol{u}$ satisfy certain nonlocal compatibility conditions at the initial time, the solution of the Navier-Stokes equations cannot be expected to have bounded derivatives of order higher than two up to the initial time, and indeed, for $\boldsymbol{u}(0) \in H^{s}(\Omega)^{d}$, with $s \geq 2$ and $\boldsymbol{f}_{\boldsymbol{u}}$ sufficiently regular, one can only expect $\boldsymbol{u} \in L^{\infty}\left(0, T, H^{2}\right) \cap L^{2}\left(0, T, H^{3}\right)$ and $\boldsymbol{u}_{t} \in L^{\infty}\left(0, T, L^{2}\right) \cap L^{2}\left(0, T, H^{1}\right)$ (see e. g., [13]). In this case, the analysis in the present section shows that one can expect $O(h)$ and $O\left(h^{2}\right)$ errors for elements of degree $k \geq 1$ and $k \geq 2$, respectively. Notice however that the above-mentioned nonlocal compatibility conditions are indeed satisfied if the initial velocity is on a periodic orbit or in an invariant torus, a very common situation in practical numerical studies of thermal convection problems modelled by (1), [14, [17.

\subsection{Error bound for the pressure}

For the error bound of the pressure we follow the error analysis in 10. Take $\boldsymbol{v}=$ $\boldsymbol{v}_{h} \in V_{h}$ in the first equation in (5), subtract from $(26)$, add $\pm\left(P_{h}^{0} p, \nabla \cdot \boldsymbol{v}_{h}\right)$ and apply the inf-sup condition (9) so that after some rearrangements we get

$$
\begin{aligned}
\beta_{0}\left\|p_{h}-P_{h}^{0} p\right\|_{0} & \leq \varepsilon\left\|\nabla\left(\boldsymbol{u}_{h}-\boldsymbol{u}\right)\right\|_{0}+\left\|B\left(\boldsymbol{u}_{h}, \boldsymbol{u}_{h}\right)-B(\boldsymbol{u}, \boldsymbol{u})\right\|_{-1} \\
& +\left\|\partial_{t} \boldsymbol{e}_{h}\right\|_{-1}+\mu\left\|\nabla \cdot\left(\boldsymbol{u}-\boldsymbol{u}_{h}\right)\right\|_{0}+\left\|\partial_{t} \boldsymbol{\tau}_{1}\right\|_{-1} \\
& +\left\|\tau_{3}\right\|_{0}+\left\|\theta_{h}-\theta\right\|_{-1}
\end{aligned}
$$

We observe that the terms $\varepsilon\left\|\nabla\left(\boldsymbol{u}_{h}-\boldsymbol{u}\right)\right\|_{0}$ and $\mu\left\|\nabla \cdot\left(\boldsymbol{u}-\boldsymbol{u}_{h}\right)\right\|_{0}$ are bounded due to Theorem 2, This is also the case of the last term on the right-hand side of (50) if we take into account that $\left\|\theta_{h}-\theta\right\|_{-1} \leq\left\|\theta_{h}-\theta\right\|_{0}$. The same applies to $\left\|\partial_{t} \boldsymbol{\tau}_{1}\right\|_{-1}$ using estimate (47) The term $\left\|\tau_{3}\right\|_{0}=\left\|P_{h}^{0} p-p\right\|_{0}$ is estimated in 12p. For the second term on the right-hand side of $(50)$ we argue by duality. For $\varphi \in H_{0}^{1}(\Omega)^{d}$, using the skew symmetry property (28) we write

$$
\left(B\left(\boldsymbol{u}_{h}, \boldsymbol{u}_{h}\right)-B(\boldsymbol{u}, \boldsymbol{u}), \boldsymbol{\varphi}\right)=-b\left(\boldsymbol{u}_{h}-\boldsymbol{u}, \boldsymbol{\varphi}, \boldsymbol{u}_{h}\right)-b\left(\boldsymbol{u}, \boldsymbol{\varphi}, \boldsymbol{u}_{h}-\boldsymbol{u}\right),
$$

so that applying Hölder's inequality we may write

$$
\begin{aligned}
\left|\left(B\left(\boldsymbol{u}_{h}, \boldsymbol{u}_{h}\right)-B(\boldsymbol{u}, \boldsymbol{u}), \boldsymbol{\varphi}\right)\right| \leq & \left\|\boldsymbol{u}_{h}-\boldsymbol{u}\right\|_{L^{p}}\|\nabla \boldsymbol{\varphi}\|_{0}\left\|\boldsymbol{u}_{h}\right\|_{L^{q}} \\
& +\left\|\nabla \cdot\left(\boldsymbol{u}_{h}-\boldsymbol{u}\right)\right\|_{0}\|\boldsymbol{\varphi}\|_{L^{2 d}}\left\|\boldsymbol{u}_{h}\right\|_{L^{2 d /(d-1)}} \\
& +\|\boldsymbol{u}\|_{\infty}\|\nabla \boldsymbol{\varphi}\|_{0}\left\|\boldsymbol{u}_{h}-\boldsymbol{u}\right\|_{0} .
\end{aligned}
$$

where $\frac{1}{p}+\frac{1}{q}=\frac{1}{2}$. Since by Sobolev's inequality (6) we have $\|\boldsymbol{\varphi}\|_{L^{2 d}} \leq\|\boldsymbol{\varphi}\|_{1}$, it follows that

$$
\begin{gathered}
\left\|B\left(\boldsymbol{u}_{h}, \boldsymbol{u}_{h}\right)-B(\boldsymbol{u}, \boldsymbol{u})\right\|_{-1} \leq \\
+\left\|\boldsymbol{u}_{h}\right\|_{L^{q}}\left\|\boldsymbol{u}_{h}-\boldsymbol{u}\right\|_{L^{p}}+\left\|\nabla \cdot\left(\boldsymbol{u}_{h}-\boldsymbol{u}\right)\right\|_{0}\|\| \boldsymbol{u}_{h} \|_{L^{2 d /(d-1)}} \\
+\|\boldsymbol{u}\|_{\infty}\left\|\boldsymbol{u}_{h}-\boldsymbol{u}\right\|_{0} .
\end{gathered}
$$


By writing $\boldsymbol{u}_{h}=\left(\boldsymbol{u}_{h}-\hat{\boldsymbol{u}}_{h}\right)+\hat{\boldsymbol{u}}_{h}$ and applying the inverse inequality (8) Sobolev's inequality (6) and standard interpolation estimates we have

$$
\left\|\boldsymbol{u}_{h}\right\|_{L^{2 d /(d-1)}} \leq C h^{-1 / 2}\left\|\boldsymbol{e}_{h}\right\|_{0}+C\left\|\hat{\boldsymbol{u}}_{h}\right\|_{1 / 2} \leq C h^{-1 / 2}\left\|\boldsymbol{e}_{h}\right\|_{0}+C\left(\left\|\hat{\boldsymbol{u}}_{h}\right\|_{0}\left\|\hat{\boldsymbol{u}}_{h}\right\|_{1}\right)^{1 / 2} .
$$

For $k \geq 2$ we write $\left\|\hat{\boldsymbol{u}}_{h}\right\|_{0}\left\|\hat{\boldsymbol{u}}_{h}\right\|_{1} \leq\|\boldsymbol{u}\|_{0}\left(\left\|\hat{\boldsymbol{u}}_{h}-\boldsymbol{u}\right\|_{1}+\|\boldsymbol{u}\|_{1}\right) \leq C\|\boldsymbol{u}\|_{0}\|\boldsymbol{u}\|_{1}$ where in the last inequality we have applied (13) with $j=0$ and $m=1$. For $k=1$ we write $\left\|\hat{\boldsymbol{u}}_{h}\right\|_{0}\left\|\hat{\boldsymbol{u}}_{h}\right\|_{1} \leq\left(\left\|\hat{\boldsymbol{u}}_{h}-\boldsymbol{u}\right\|_{0}+\|\boldsymbol{u}\|_{0}\right)\|\boldsymbol{u}\|_{1} \leq C\left(\|\boldsymbol{u}\|_{1} h+\|\boldsymbol{u}\|_{0}\right)\|\boldsymbol{u}\|_{1}$, where in the last inequality we have applied (17) with $j=0$. Thus, we may write

$$
\left\|\boldsymbol{u}_{h}\right\|_{L^{2 d /(d-1)}} \leq C h^{-1 / 2}\left\|\boldsymbol{e}_{h}\right\|_{0}+\left(\left(\bar{k} h\|\boldsymbol{u}\|_{1}+\|\boldsymbol{u}\|_{0}\right)\|\boldsymbol{u}\|_{1}\right)^{1 / 2}
$$

and taking $q=2 d$ if $k=1$ and $q=\infty$ otherwise we have

$$
\left\|\boldsymbol{u}_{h}\right\|_{L^{q}} \leq \begin{cases}C h^{-(d-1) / 2}\left\|\boldsymbol{e}_{h}\right\|_{0}+C\|\boldsymbol{u}\|_{1} \leq C h^{-1}\left\|\boldsymbol{e}_{h}\right\|_{0}+C\|\boldsymbol{u}\|_{1}, & k=1 \\ C h^{-d / 2}\left\|\boldsymbol{e}_{h}\right\|_{0}+C\left(\|\boldsymbol{u}\|_{d-2}\|\boldsymbol{u}\|_{2}\right)^{1 / 2}, & k \geq 2,\end{cases}
$$

and, arguing similarly

$$
\left\|\boldsymbol{u}_{h}-\boldsymbol{u}\right\|_{L^{p}} \leq \begin{cases}C h^{-1 / 2}\left\|\boldsymbol{e}_{h}\right\|_{0}+C\|\boldsymbol{u}\|_{1} h^{1 / 2}, & k=1 \\ \left\|\boldsymbol{u}_{h}-\boldsymbol{u}\right\|_{0}, & k \geq 2 .\end{cases}
$$

Thus, we have

$$
\begin{aligned}
\left\|B\left(\boldsymbol{u}_{h}, \boldsymbol{u}_{h}\right)-B(\boldsymbol{u}, \boldsymbol{u})\right\|_{-1} & \leq C\left(h^{-d / 2}\left\|\boldsymbol{e}_{h}\right\|_{0}+\left(\|\boldsymbol{u}\|_{d-2}\|\boldsymbol{u}\|_{1}\right)^{1 / 2}\right)\left\|\boldsymbol{u}_{h}-\boldsymbol{u}\right\|_{0} \\
& \left.+C\left(h^{-1 / 2}\left\|\boldsymbol{e}_{h}\right\|_{0}+\left(\|\boldsymbol{u}\|_{d-2}\|\boldsymbol{u}\|_{1}\right)^{1 / 2}\right)\right)\left\|\nabla \cdot\left(\boldsymbol{u}_{h}-\boldsymbol{u}\right)\right\|_{0}
\end{aligned}
$$

if $k \geq 2$ and, if $k=1$,

$$
\begin{aligned}
\left\|B\left(\boldsymbol{u}_{h}, \boldsymbol{u}_{h}\right)-B(\boldsymbol{u}, \boldsymbol{u})\right\|_{-1} \leq & C\left(\|\boldsymbol{u}\|_{1}+h^{-1}\left\|\boldsymbol{e}_{h}\right\|_{0}\right) h^{-1 / 2}\left\|\boldsymbol{e}_{h}\right\|_{0} \\
& +C h^{1 / 2}\|\boldsymbol{u}\|_{1}^{2}+C h^{-1 / 2}\left\|\boldsymbol{e}_{h}\right\|_{0}\left\|\nabla \cdot\left(\boldsymbol{u}_{h}-\boldsymbol{u}\right)\right\|_{0} \\
& +C\left(\left(\|\boldsymbol{u}\|_{1} h+\|\boldsymbol{u}\|_{0}\right)\|\boldsymbol{u}\|_{1}\right)^{1 / 2}\left\|\nabla \cdot\left(\boldsymbol{u}_{h}-\boldsymbol{u}\right)\right\|_{0} \\
& +C\left(\|\boldsymbol{u}\|_{1}\|\boldsymbol{u}\|_{2}\right)^{1 / 2}\left\|\boldsymbol{u}_{h}-\boldsymbol{u}\right\|_{0}
\end{aligned}
$$

Observe that the quantities multiplying $\left\|\boldsymbol{u}_{h}-\boldsymbol{u}\right\|_{0}$ or $h^{-1 / 2}\left\|\boldsymbol{e}_{h}\right\|_{0}$ and $\left\|\nabla \cdot\left(\boldsymbol{u}_{h}-\boldsymbol{u}\right)\right\|_{0}$ in these last two estimates are bounded, either by the smoothness assumed on $\boldsymbol{u}$ or as a consequence of Theorem 2 .

To bound $\left\|\partial_{t} \boldsymbol{e}_{h}\right\|_{-1}$ we also argue as in [10]. From [3, Lemma 3.11] it holds

$$
\left\|\partial_{t} \boldsymbol{e}_{h}\right\|_{-1} \leq C h\left\|\partial_{t} \boldsymbol{e}_{h}\right\|_{0}+C\left\|A^{-1 / 2} \Pi \partial_{t} \boldsymbol{e}_{h}\right\|_{0}
$$

and from [3, (2.15)]

$$
\left\|A^{-1 / 2} \Pi \partial_{t} \boldsymbol{e}_{h}\right\|_{0} \leq C h\left\|\partial_{t} \boldsymbol{e}_{h}\right\|_{0}+\left\|A_{h}^{-1 / 2} \partial_{t} \boldsymbol{e}_{h}\right\|_{0} .
$$

Applying now (51), 52), the symmetry of the operator $A_{h}$ and inverse inequality (8) we reach

$$
\begin{aligned}
\left\|\partial_{t} \boldsymbol{e}_{h}\right\|_{-1} & \leq C h\left\|\partial_{t} \boldsymbol{e}_{h}\right\|_{0}+C\left\|A_{h}^{-1 / 2} \partial_{t} \boldsymbol{e}_{h}\right\|_{0} \\
& =C h\left\|A_{h}^{1 / 2} A_{h}^{-1 / 2} \partial_{t} \boldsymbol{e}_{h}\right\|_{0}+C\left\|A_{h}^{-1 / 2} \partial_{t} \boldsymbol{e}_{h}\right\|_{0} \\
& =C h \mid \nabla\left(A_{h}^{-1 / 2} \partial_{t} \boldsymbol{e}_{h}\right)\left\|_{0}+C\right\| A_{h}^{-1 / 2} \partial_{t} \boldsymbol{e}_{h} \|_{0} \\
& \leq C\left\|A_{h}^{-1 / 2} \partial_{t} \boldsymbol{e}_{h}\right\|_{0} .
\end{aligned}
$$


Taking into account that $\left\|A_{h}^{-1 / 2} \Pi_{h}^{\mathrm{div}} \mathbf{g}\right\|_{0} \leq\|\mathbf{g}\|_{-1}$, for all $\mathbf{g} \in L^{2}(\Omega)^{d}$, see [3, (2.16)] and arguing as in [10] we get

$$
\begin{aligned}
\left\|A_{h}^{-1 / 2} \partial_{t} \boldsymbol{e}_{h}\right\|_{0} & \leq \varepsilon\left\|\nabla \boldsymbol{e}_{h}\right\|_{0}+\left\|B\left(\boldsymbol{u}_{h}, \boldsymbol{u}_{h}\right)-B(\boldsymbol{u}, \boldsymbol{u})\right\|_{-1}+C \mu\left\|\nabla \cdot\left(\boldsymbol{u}_{h}-\boldsymbol{u}\right)\right\|_{0} \\
& +(1-\bar{k}) \varepsilon\left\|\nabla\left(\hat{\boldsymbol{u}}_{h}-\boldsymbol{u}\right)\right\|_{0}+\bar{k}\left\|\partial_{t} \boldsymbol{\tau}_{1}\right\|_{-1}+C\left\|\tau_{3}\right\|_{0} \\
& +C\left\|\theta-\theta_{h}\right\|_{-1} .
\end{aligned}
$$

Observe that all the the terms on the right-hand side of (53) are also present on the right-hand side of (50) except $\varepsilon\left\|\nabla \boldsymbol{e}_{h}\right\|_{0}$ and $\varepsilon\left\|\nabla\left(\hat{\boldsymbol{u}}_{h}-\boldsymbol{u}\right)\right\|_{0}$ which are estimated in Theorem 2 and $\sqrt{13})$, respectively. Finally, by the splitting $p_{h}-p=\left(p_{h}-P_{h}^{0} p\right)+$ $\left(P_{h}^{0} p-p\right)$ we arrive to the following result

Theorem 3 Under the assumptions of Theorem 2 and assuming also that $\partial_{t} \boldsymbol{u} \in$ $L^{2}\left(0, T, H^{k}(\Omega)^{d}\right)$, there exists a positive constant $C$ such that the following bound holds

$$
\beta_{0}^{2} \int_{0}^{T}\left\|\left(p_{h}-p\right)(t)\right\|_{0}^{2} d t \leq C h^{2 k-\bar{k}}
$$

\section{References}

[1] Robert A. Adams. Sobolev spaces. Academic Press [A subsidiary of Harcourt Brace Jovanovich, Publishers], New York-London, 1975. Pure and Applied Mathematics, Vol. 65.

[2] S. Agmon, Lectures on Elliptic Boundary Value Problems. Prepared for publication by B. Frank Jones, Jr. with the assistance of George W. Batten, Jr. Revised edition of the 1965 original. AMS Chelsea Publishing, Providence, RI, 2010.

[3] Blanca Ayuso, Bosco García-Archilla, and Julia Novo. The postprocessed mixed finite-element method for the Navier-Stokes equations. SIAM J. Numer. Anal., 43(3):1091-1111, 2005.

[4] Philippe G. Ciarlet. The finite element method for elliptic problems. NorthHolland Publishing Co., Amsterdam, 1978. Studies in Mathematics and its Applications, Vol. 4

[5] T. Chacón Rebollo, M. Gómez Mármol, F. Hecht, S. Rubino and I. Sánchez Muñoz. A High-Order Local Projection Stabilization Method for Natural Convection Problems. J. Sci. Comput. 74(2):667-692, 2018.

[6] Hongsen Chen. Pointwise error estimates for finite element solutions of the Stokes problem. SIAM J. Numer. Anal., 44(1):1-28, 2006.

[7] P. Clément. Approximation by finite element functions using local regularization. RAIRO Anal. Numér., 9:77-84, 1975.

[8] P. Constantin \& C. Foias, Navier-Stokes Equations, Chicago Lectures in Mathematics. University of Chicago Press, Chicago, IL, 1988.

[9] H. Dallmann and D. Arndt. Stabilized Finite Element Methods for the Oberbeck-Boussinesq Model. J. Sci. Comput, 69:244-273, 2016. 
[10] J. de Frutos, B. García-Archilla, V. John and J. Novo. Analysis of the graddiv stabilization for the time-dependent Navier-Stokes equations with inf-sup stable finite elements, Adv. Comput. Math. 44:195-225, 2018.

[11] J. de Frutos, B. García-Archilla and J. Novo. Error Analysis of Projection Methods for Non inf-sup Stable Mixed Finite Elements: The Navier-Stokes Equations, J. Sci. Comput., 74:426-455,2018.

[12] V. John. Finite element methods for incompressible flow problems. Springer Series in Computational Mathematics vol. 51 of Springer Series in Computational Mathematics. Springer, Cham (2016).

[13] John G. Heywood and Rolf Rannacher. Finite element approximation of the nonstationary Navier-Stokes problem. I. Regularity of solutions and secondorder error estimates for spatial discretization. SIAM J. Numer. Anal., 19(2):275-311, 1982.

[14] M. Net \& J. Sánchez Umbría, Periodic orbits in tall laterally heated rectangular cavities, Phys. Rev. E, 95, 023102 (2017)

[15] P. W. Schoroeder and G. Lube. Stabilised dG-FEM for incompressible natural convection flows with boundary and moving interior layers on non-adapted meshes. J. Comput. Phys. 335:760-779, 2017.

[16] A. H. Schatz and L. B. Wahlbin. On the quasi-optimality in $L^{\infty}$ of the $H^{1}$ projection into finite-element spaces. Math. Comp. 38:1-21 (19820.

[17] S. Xin \& P. Le Quéré, An extended Chebyshev pseudo-spectral benchmark for the 8:1 differentially heated cavity, Int. J. Numer. Meth. Fluids, 40 (2002), 981-998. 